\title{
Laboratory Spectroscopy of PAHs
}

\author{
T. Pino ${ }^{1}$, G. Féraud ${ }^{2}$, Ph. Bréchignac ${ }^{1}$, E. J. Bieske ${ }^{3}$ \\ and T. W. Schmidt ${ }^{4}$ \\ ${ }^{1}$ Institut des Sciences Moléculaire d'Orsay, CNRS, Univ. Paris Sud, 91405 Orsay, France \\ email: thomas.pino@u-psud.fr \\ ${ }^{2}$ Physique des Intéractions ioniques et Moléculaires, CNRS, Univ. Aix Marseilles, \\ 13397 Marseilles, France \\ ${ }^{3}$ School of Chemistry, University of Melbourne, 3010 Victoria, Australia \\ ${ }^{4}$ School of Chemistry, The University of Sydney, NSW 2006, Australia
}

\begin{abstract}
Spectroscopic investigations of PAHs have been conducted for many years, commencing with solid and solution studies and more recently including gas phase characterisation of a broad range of different species. Through the development of new, efficient methods of production and more sensitive spectroscopic techniques, fresh data are becoming available for not only neutral species, but also for radicals, ions and clusters.
\end{abstract}

Keywords. PAHs, electronic spectroscopy, photodissociation, astrochemistry

\section{Introduction}

PAHs are a family of molecules that exhibit enormous structural and isomeric variation; even with 10 or fewer aromatic cycles in the carbon skeleton more than 10000 species can be constructed, even without the inclusion of substituents, consideration of the charge states, or introduction of radical derivatives. Examining their relevance to the diffuse interstellar bands spectrum would therefore rapidly becomes a hopeless task when adding astrophysical constraints: their electronic spectra should be measured for cold and isolated PAHs, and these molecules are often difficult to introduce into the gas phase. For example hexabenzocoronene (HBC) melts before it can be vaporised. However, evidence that related molecules are present in space is strong due to the aromatic infrared bands (Léger \& Puget 1984, Allamandola et al. 1985), thus their possible contributions to the interstellar absorption curve have to be taken into account. Although the spectral band positions and shapes are unique for a given molecule, sub-families can be identified and trends can be drawn. In particular the connection between the molecular structure (compact, peri-condensed, edge effect, all-benzenoid structures) and the electronic structure can be studied and used to select promising PAHs. The wavelength range, intensities and line widths of the lower electronic transitions can thus be roughly predicted in order to determine the pertinence of a given sub-family. Effects of the charge states, hydrogenation, substitution (and others) can be studied for a few species and deduced for the other members of a sub-family. By these means, far from being hopeless, electronic spectroscopy of PAHs brings important information that helps narrow the range of candidates for DIB carriers.

To attain such understanding requires concerted experimental and theoretical efforts. Extension of the knowledge beyond "standard" PAHs is in progress thanks to new production methods and spectroscopic techniques. In fact, it is mainly because of laboratory astrophysics that gas phase PAHs are currently being studied, with the information also 
serving combustion science. This is an interesting connection considering how our understanding of combustion chemistry has fertilised progress in astrochemistry.

In this review we focus on selected recent activities (after 2010) to illustrate progress in the laboratory characterisation of PAHs. Previous reviews cover earlier work (Salama et al. 2007, Pino et al. 2011). The paper provides a tour through the electronic spectroscopy of PAHs according to charge state, and by radical or substitution effects. One may note that anions remain barely explored with only matrix data being available. Thus, only neutral and cation PAHs are covered in this work.

\section{Neutral PAHs}

\subsection{Production of PAHs in chemical reactors}

PAHs have been introduced into the gas phase from chemically synthesised samples using their natural vapour pressure or through laser ablation (Simpson et al. 2002, Kokkin et al. 2008). An alternative, that becomes increasingly necessary for larger species is to synthesise the PAH molecules in the gas phase using, for example, incomplete combustion in a gas phase reactor. Flames, plasmas, (laser) pyrolysis are other reactive media that can in principle produce sufficient concentrations of PAHs for gas phase experiments. However, coupling the chemical reactor with laser spectroscopic techniques is not straightforward because usually further cooling is required before laser interrogation. Radicals produced from an electric discharge in a supersonic expansion can also be probed directly in the expansion zone (see the next section). PAHs produced in rich flames were subsequently cooled and then probed using resonant 2-photon ionization spectroscopy (Carpentier et al. 2013). These promising methods await extension to larger PAHs and more efficient cooling after sampling from the reactor.

\subsection{High resolution spectroscopy}

Recent years have seen numerous high resolution studies on PAHs, notably from the group of Masaaki Baba in Kyōto. Ultrahigh resolution spectroscopy of benzene and naphthalene have afforded structural parameters to less than a $\mathrm{m} \AA$ (Baba et al. 2011), and a similar study on perylene has resulted in rotational constants determined to $10^{-5} \mathrm{~cm}^{-1}$ precision (Kowaka et al. 2010). This latter study shows the very small geometry change between the ground and excited states of perylene, to which its high fluorescence yield is attributed. Where internal conversion does occur, it is determined to be promoted by a single mode (Suganuma et al. 2011). Indeed, studies of mode-selectivity in the internal conversion of pyrene and perylene in the gas phase have added valuable data to be consumed by those concerned with non-adiabatic calculations on large molecules, on the frontiers of molecular physics (Kowaka et al. 2012). Pyrene has also been studied by McCall and co-workers (Brumfield et al. 2012), who determined it to be the most rigid rotor yet observed. The resolution of this study was about $10^{-7} \mathrm{~cm}^{-1}$. Phenanthrene and anthracene have also been studied by ultrahigh resolution, revealing a normal deuterium effect for phenanthrene and a reverse effect (deuterium with shorter fluorescence lifetime) for anthracene (Kowaka et al. 2012).

\subsection{Neutral radicals}

It has long been known that many PAH neutral radicals present their lowest electronic transitions in the visible region. This may be understood in terms of a radical electron level being introduced into the "bandgap" generated by the parent PAH system. After mixing between the electronic levels, the radical absorbs at a little more than half the 


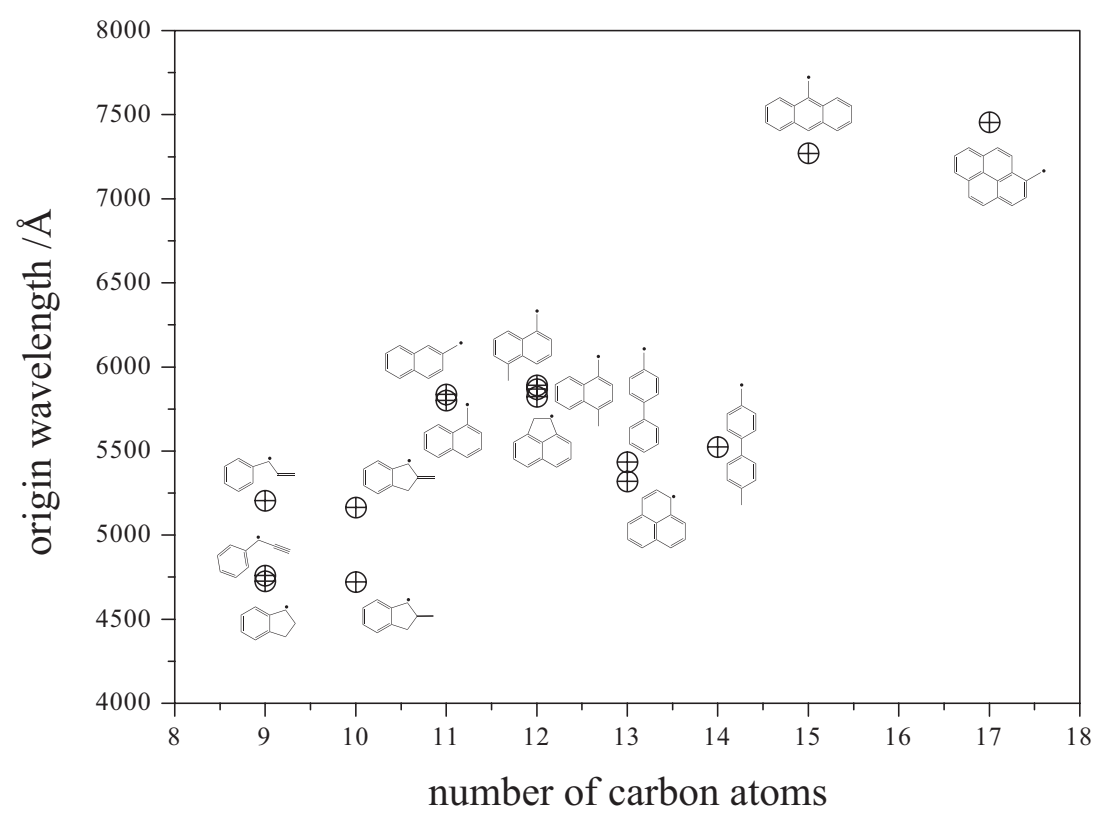

Figure 1. The origin positions (in $\AA$ ), of PAH radicals detected in the Sydney laboratory. There is a general trend to longer wavelength with increasing size, as the $\pi$ system grows.

energy of the parent. As such, while benzene absorbs near $2600 \AA$, benzyl radical absorbs at $4600 \AA$ (Fukushima \& Obi 1990). Similarly, naphthalene absorbs at $3100 \AA$, and naphthylmethyl radical absorbs near $5800 \AA$ (Chalyavi et al. 2011). Since these electronic transitions are to the lowest electronic state, they remain sharp, and are not broadened by lifetime effects as observed for the radical cations. Indeed, for most of the species, fluorescence is observed.

The Sydney laboratory has generated the spectra of many PAH radicals in recent years (Troy et al. 2009, Chalyavi et al. 2011, 2011, 2012, Reilly et al. 2008, O'Connor et al.2013), with the largest species comprising 17 carbon atoms: the pyrenylmethyl radical (O'Connor et al. 2013). The absorption positions, none of which match a diffuse interstellar band, are summarised in Fig. 1. Since the absorption positions are governed mostly by the size and connectivity of the $\pi$-system, we find that the indanyl and 2methylindanyl radicals both absorb near $4700 \AA$ (Troy et al. 2009). Likewise, all four radicals in Fig. 1 built on the 11-p-orbital naphthylmethyl chromophore absorb near $5800 \AA$ (Chalyavi et al. 2011). Since these species also emit fluorescence, there remains a possibility that they are involved in the unidentified emission from the Red Rectangle, a mysterious proto-planetary nebula.

All of the $D_{1} \leftarrow D_{0}$ transitions of odd-alternant PAH radicals are weak. As such, it is rather unlikely that they would be easily detected astronomically. This is a consequence of the pairing principle (Coulson \& Rushbrooke 1940), and, as explained by Longuet-Higgins \& Pople (1955), is due to mixing between the two degenerate one-electron transitions into and out of the singly occupied orbital. One might naïvely expect that the other combination, which carries the oscillator strength, will be observable. Our initial theoretical investigations reveal that further mixing between excited configurations occurs, and that the picture is far from simple for most chromophores. Yet, we hope to observe the $D_{2} \leftarrow D_{0}$ transition of phenalenyl in the near future. Hole-burning spectroscopy has revealed that the strong $D_{n} \leftarrow D_{0}$ absorptions of naphthylmethyl radical, for instance, 
are time-uncertainty broadened. A Lorentzian fit to these bands suggests a lifetime of the order $50 \mathrm{fs}$, and a bandwidth inconsistent with most DIBs (Woodhouse 2012).

So, what is left to do with PAH radicals? We know that the lower transitions are too weak, and the higher transitions are too broad. By 17 or so carbons in the $\pi$-system, the $D_{1} \leftarrow D_{0}$ transition is exiting the visible region at the low energy end (O'Connor et al. 2013). It seems at this stage extremely unlikely that these radicals can be responsible for the DIBs. However, their IEs are very low, and were such species to be ionized in the ISM, they would yield a closed-shell cation, also absorbing in the visible region, and often blueshifted as compared to the cation. Closed-shell cation spectra are emerging from various laboratories, notably those of Jouvet, Pino, Bieske and Maier (vide infra). Additionally, the Sydney laboratory has applied a two-laser-pulse sequence to produce $0 \mathrm{~K}$ cations from PAH radicals which have then been subjected to the dissociation spectroscopy pioneered by Jouvet. Such experiments are to be published shortly (Krechkivska et al. 2013).

\section{PAH cations}

\subsection{Spectroscopy of ionic PAHs}

Deciding whether polycyclic aromatic hydrocarbons and related species [polycyclic aromatic nitrogen heterocycles (PANHs)] play a role in interstellar space requires laboratory spectra that can be compared with astronomical data. A match between molecular transition energies and intensities measured in the laboratory and astronomical data is a necessary condition for the species to exist in space. Given that there is a poor correlation in intensities between most DIBs along different lines of sight, it has been proposed that any molecular carrier should have an electronic spectrum dominated by its origin transition. Therefore, the intensity distribution of even a relatively low resolution laboratory spectrum can provide a guide as to whether or not a particular species is a candidate as DIB carrier.

At Melbourne electronic spectra are recorded using resonance-enhanced photodissociation of $\mathrm{He}$ and Ar tagged PAHs (Dryza et al. 2012a,b). The $\mathrm{PAH}^{+}-\mathrm{Ar}$ and $\mathrm{PAH}^{+}-\mathrm{He}$ complexes are produced in a pulsed supersonic expansion of either helium or argon gas seeded with the neutral PAH vapour, crossed in the early stages by an electron beam. The complexes are mass selected in a quadrupole mass filter and then exposed to a tunable light beam in an octopole ion guide. Photodissociation (loss of the rare gas atom) occurs when the light is resonant with a vibronic transition of the weakly-bound complex. Loss of the rare gas atom(s) and production of photofragment $\mathrm{PAH}^{+}$ion is monitored as a function of wavelength to give the action spectrum.

This approach has been used to obtain electronic spectra of several Ar-tagged aromatic cations, including naphthalene ${ }^{+}$, quinoline $^{+}$, isoquinoline ${ }^{+}$(Dryza et al. 2012a), benzylium $^{+}$(Dryza et al. 2012b), and indene ${ }^{+}$(Chalyavi et al. 2013). Although the vibronic bands of the molecular cations measured in this way are usually broader than those of complexes formed using REMPI ( $\Delta \tilde{\nu}$ of $80 \mathrm{vs} 20 \mathrm{~cm}^{-1}$ ), the advantage is that one can readily form the Ar-tagged and He-tagged PAH cations without complicated REMPI schemes. This makes the approach suitable for survey scans, akin to matrix isolation spectroscopy but with narrower bands and smaller band shifts. The band broadening is presumably associated with the complexes possessing vibrational energy, particularly in the intermolecular modes, so that the observed peaks consist of overlapping hot bands. This can also lead to slight shifts in the band centres.

As an example, Fig. 2 compares spectra of the isoelectronic naphthalene ${ }^{+}-$Ar, $_{\text {quinoline }}{ }^{-}$ Ar and isoquinoline ${ }^{+}$-Ar complexes. Spectra of the three molecular cations are similar, 


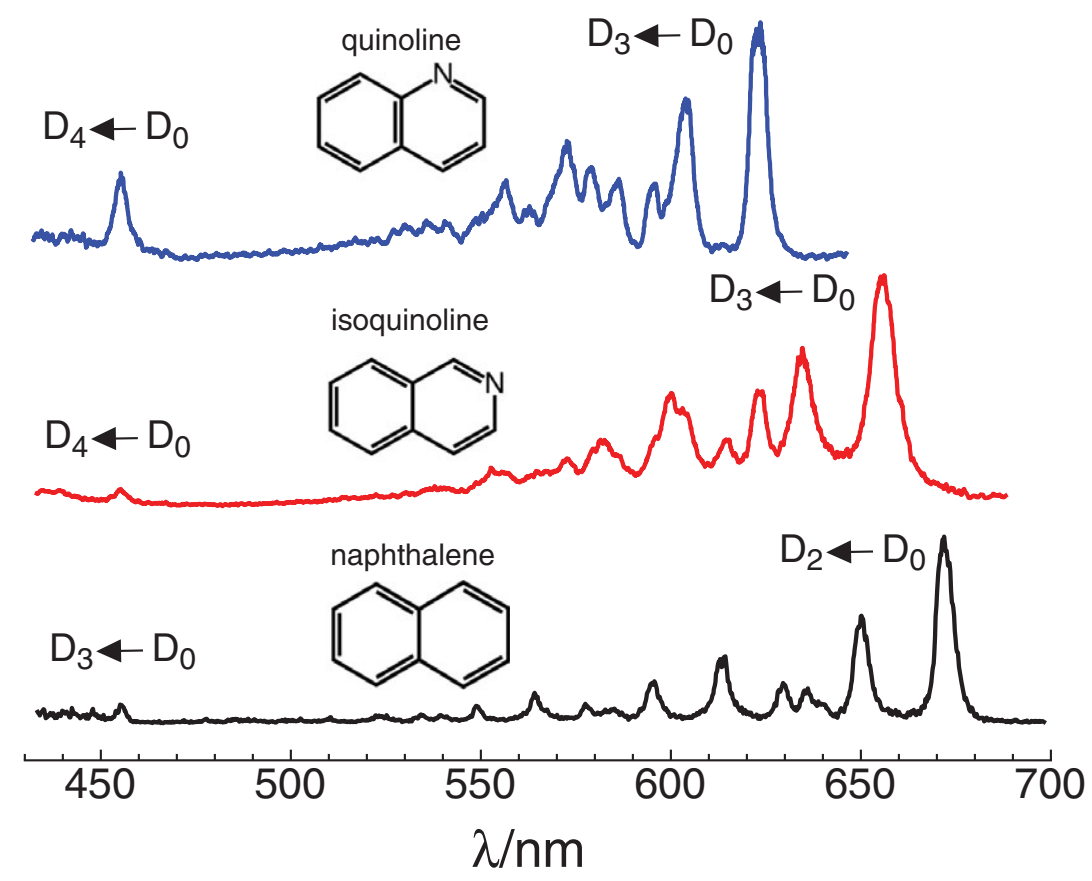

Figure 2. Electronic spectra of naphthalene ${ }^{+}-\operatorname{Ar}$ (bottom), isoquinoline ${ }^{+}-\operatorname{Ar}$ (middle), and quinoline $^{+}-\mathrm{Ar}$ (top) complexes, recorded by monitoring the Ar loss channel.

and consist of a band system with an intense origin transition at around $650 \mathrm{~nm}$, accompanied by progressions extending to roughly $520 \mathrm{~nm}$, and a second weaker system with a prominent, lone origin band transition near $455 \mathrm{~nm}$. The vibronic structure of visible systems are assigned by comparison with simulated spectra based on time-dependent density functional theory calculations (Dryza et al. 2012). The naphthalene ${ }^{+}-$Ar photodissociation spectrum is similar to previous gas-phase spectra (Pino et al. 1999, Biennier et al. 2003) and matrix isolation spectra (Salama \& Allamandola 1991), and is dominated by the $\mathrm{D}_{2} \leftarrow \mathrm{D}_{0}$ band system, with an intense origin transition at $14873 \pm 15 \mathrm{~cm}^{-1}$, accompanied by several strong progressions. In contrast, the $\mathrm{D}_{3} \leftarrow \mathrm{D}_{0}$ band is comparatively weak, and only the origin peak at $21959 \pm 15 \mathrm{~cm}^{-1}$ is obvious.

The PANH cations isoquinoline ${ }^{+}$and quinoline ${ }^{+}$also have absorptions in the visible part of the spectrum that could conceivably correspond to DIBs. Obviously, it is difficult to make definite matches between the isoquinoline ${ }^{+}$and quinoline ${ }^{+}$transitions and astronomical absorptions because of shifts associated with the attached argon atom(s), and because the observed bands are relatively broad. However, the existence of long, strong progressions in the $D_{3} \leftarrow D_{0}$ visible band systems of isoquinoline ${ }^{+}$and quinoline ${ }^{+}$ counts against these PANH cations being DIB carriers because of the poor correlation between the strengths of any pair of DIBs collected from different ISM regions. Another molecular cation that can probably be eliminated as a DIB carrier because of its intensity distribution is the benzylium cation whose spectrum is shown in Fig. 3.

\subsection{Spectroscopy of protonated PAHs}

Protonated molecules are closed-shell cations, so their electronic structure is expected at first to be similar to that of neutral molecules. However, their electronic spectra are different (origin position, excited vibrational modes). Only few experiments exist on the 


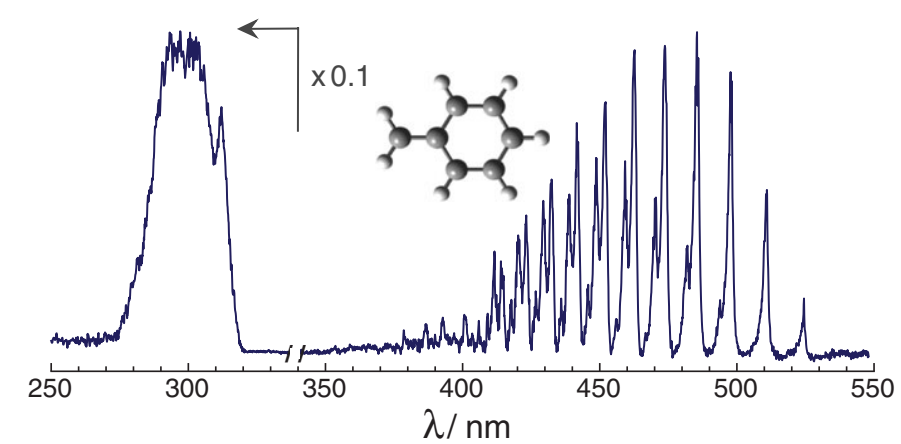

Figure 3. Electronic spectrum of the benzylium ${ }^{+}-\mathrm{Ar}$ complex recorded by monitoring the $\mathrm{Ar}$ loss channel.

electronic spectroscopy of protonated PAHs, either in matrix (Garkusha et al. 2011a,b, 2012, 2013, Naby et al. 2011) or in gas phase (Chakraborty et al. 2009, Alata et al. 2010, 2011,2012 ). In the gas phase experiment, protonation is achieved in an electrical discharge and the ions are cooled in a supersonic expansion. The ions are spectroscopically probed using photodissociation in a reflectron time-of-flight spectrometer (Fig. 4). After a first field-free region, protonated PAHs interact with the laser, then the neutral products are detected (first detector) and the ion parent and ionic fragments are reflected toward a second detector. The absorption spectrum is obtained by plotting the neutral photofragment signal as a function of excitation wavelength. Another way of producing cold and gas phase protonated PAHs is from an electrospray source coupled with a cryogenicallycooled ion trap (Alata et al. 2013) and the detection rely on the detection of the fragment ion. Electronic structure calculations (time-dependent density functional theory calculations, TD-DFT (Garkusha et al. 2011) and second-order approximate coupled-cluster calculation, RI-CC2 (Garkusha et al. 2011b, Alata et al. 2010b) performed on protonated PAHs provide information on the geometry of their ground and electronic excited states, energies of $\mathrm{S}_{n} \leftarrow \mathrm{S}_{0}(\mathrm{n}=1,2,3 \ldots)$ transitions and even the vibronic structure of electronic transitions.

Alata et al. (2010b) found that the origins of the electronic transition of protonated PAHs are redshifted with respect to that of neutral homologues (Table 1). More precisely, this redshift is not constant from one molecule to another (Table 1): it is larger for naphthalene (even number of cycles) than for anthracene (odd number of cycles). The authors explain these specificities using ab initio calculations. Electronic transitions of PAHs with an even number of cycles have a charge transfer character: one electron of the non-protonated cycle goes to the protonated cycle. This is similar to the charge transfer from non-protonated benzene to protonated benzene of the benzene dimer (Chakraborty et al. 2009) in which the absorption is in the visible, whereas it is in the UV for the neutral dimer. Electronic transitions of protonated PAHs with an odd number of cycles do not have charge transfer character, so they are closer to the neutral transition. The redshift of electronic transitions of protonated species with respect to neutral species is thus governed by the nature of orbitals involved in the transition.

Other specificities are found in the analysis of vibronic structures of protonated aromatic molecules (Fig. 4). Protonated benzene exhibits a very broad and featureless electronic transition (Chakraborty et al. 2009). This indicates an important change of geometry between the two states and a very short lifetime for the excited state due a conical intersection (Rode et al. 2009). On the contrary, protonated naphthalene, anthracene and tetracene show clear vibrational progressions, indicative of a smaller change of geometry 


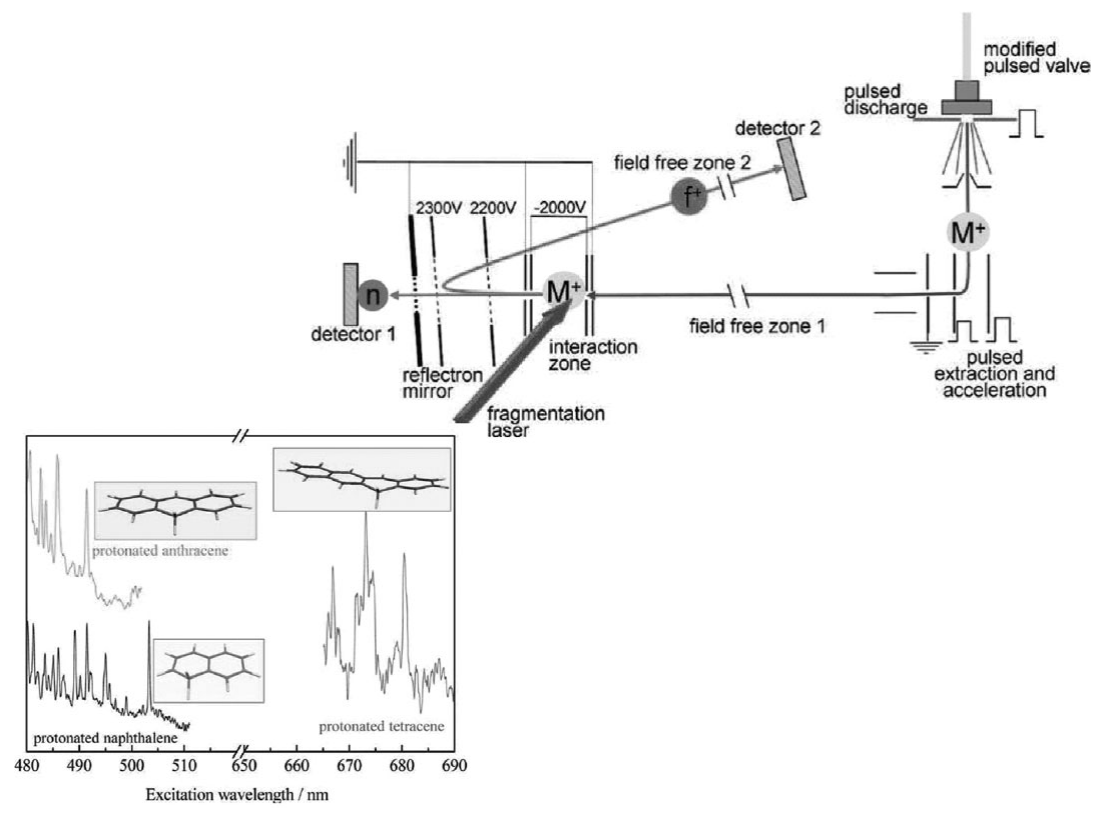

Figure 4. Scheme for the gas phase spectroscopic studies of protonated PAHs. The ions are produced in an electrical discharge, and are cooled in a supersonic expansion before being spectroscopically probed using photodissociation in a reflectron time-of-flight spectrometer. Spectra of protonated naphthalene, anthracene and tetracene. Source: Alata et al. (2010).

\begin{tabular}{lccc}
\hline molecule & neutral & protonated & vib.struct. \\
\hline benzene & $2674 \AA(4.64 \mathrm{eV})$ & $3700 \AA(3.35 \mathrm{eV})$ & no \\
naphthalene & $3120 \AA(3.97 \mathrm{eV})$ & $5034 \AA(2.46 \mathrm{eV})$ & yes \\
anthracene & $3610 \AA(3.44 \mathrm{eV})$ & $4914 \AA(2.53 \mathrm{eV})$ & yes \\
tetracene & $4965 \AA(2.50 \mathrm{eV})$ & $6799 \AA(1.82 \mathrm{eV})$ & yes \\
\hline
\end{tabular}

Table 1. Experimental values of the origins of the $\mathrm{S}_{1} \leftarrow \mathrm{S}_{0}$ transition of neutral and protonated benzene, naphthalene, anthracene and tetracene. Source: Alata et al. $(2010 \mathrm{a}, \mathrm{b})$ and references therein.

(Fig. 4)(Alata et al. 2010a,b). However the charge transfer character of the excited state leads to a larger geometry change between ground and excited state as compared to the neutral molecule responsible for the activity of modes elsewhere inactive in neutral molecules.

Several isomers can be observed for protonated fluorene (Alata et al. 2012) and indole (Alata et al. 2013), with overlapping spectra for the former or with a separation of $1 \mathrm{eV}$ for the latter.

\subsection{Spectroscopy of ionic clusters PAHs}

Resonance-enhanced photodissociation has also enabled to record the photodissociation spectra of small PAH homo-dimers. In this case the preparation of the cold cations is done by achieving laser photoionization (following 2-photon UV absorption) within the free jet expansion right at the exit of the nozzle, as explained in Friha et al. (2011). The use of a delay time, between the photodissociation laser pulse and the application of the extraction voltage guiding the cations to the time-of-flight mass spectrometer, allows to discriminate the different parent-fragment pairs according to their arrival time. An example is shown in 


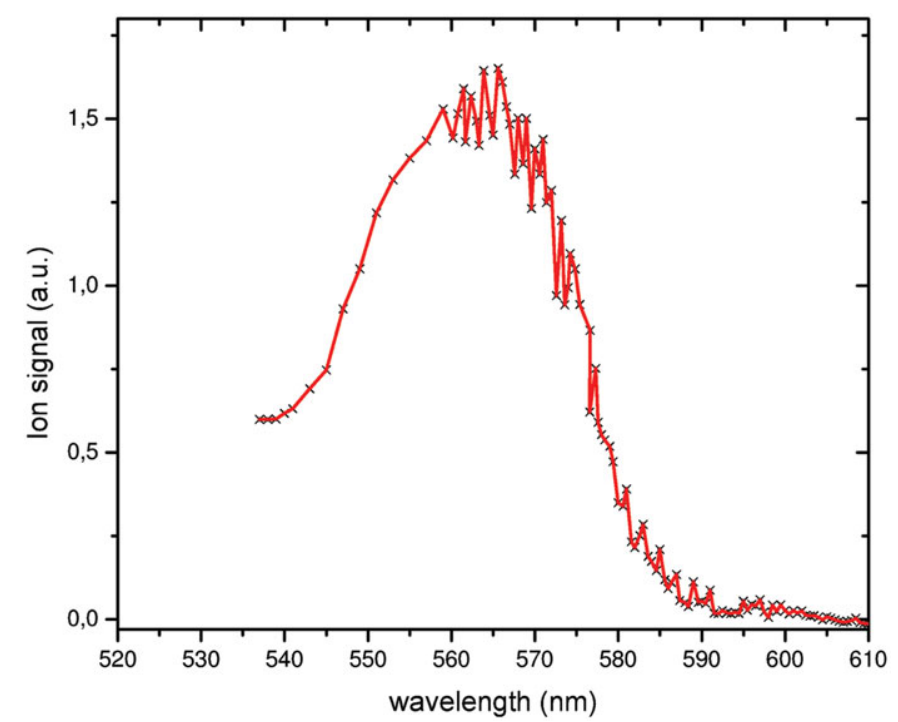

Figure 5. Visible photodissociation spectrum of the (naphthalene) $)_{2}^{+}$dimer.

Fig. 5 for the channel $\mathrm{Np}_{2}^{+} \rightarrow \mathrm{Np}^{+}+\mathrm{Np}$ of the Napthalene dimer cation. This spectrum corresponds to the absorption from the dimers ground state to its locally excited state correlating with the $\mathrm{D}_{2}$ electronic excited state in the cationic monomer. It exhibits a broad absorption band peaking near $567 \mathrm{~nm}\left(\sim 17700 \mathrm{~cm}^{-1}\right)$, to be compared with the position of the $\mathrm{D}_{2} \leftarrow \mathrm{D}_{0}$ transition in the monomer cation, i.e. $672.5 \mathrm{~nm}$, which represents a blue shift of $2840 \mathrm{~cm}^{-1}$. This shift is about $500 \mathrm{~cm}^{-1}$ larger than in the case of 2-Methylnaphthalene dimer cation (Friha et al. 2011). This difference is consistent with the existence of a steric hindering by the methyl group in a sandwich configuration of the dimer. The broadness of the visible absorption feature clearly excludes such homogeneous PAH clusters as candidates to the DIB carriers. However, clusters of mixed PAHs may not show up such large vibronic bands and laboratory spectra are to be measured to answer such question.

\section{Emission spectroscopy of non fluorescent electronic excited states of PAHs}

Rapid non-radiative relaxation often dominates for higher excited electronic states and transitions to these states appear spectrally broadened in absorption. As the gap between the ground and the first electronic states diminishes upon ionization or formation of radical structures, most ions do not fluoresce and display broadened bands. This can be rationalised in the form of the energy gap law (Pino et al. 2011), even if for most small gaps a conical intersection can be found (Tokmachev et al. 2010). However, when long time-scale dynamics can be accessed, i.e. longer than the electronic relaxation time-scale of the excited state, fluorescence can again be observed. Effectively when inverse internal conversion occurs, anomalous or so-called Poincaré fluorescence takes place. In all cases infrared emission occurs and in fact is at the core of the PAH hypothesis in astrophysics (Léger \& Puget 1984, Allamandola et al. 1985). Thus, the electronic transition to nonfluorescing states can also be measured by emission spectroscopy. 


\subsection{Laser induced infrared fluorescence}

Infrared fluorescence (IRF) proceeds after excitation to a rapidly relaxing electronic state, which converts electronic energy into vibrational energy. Under sufficiently isolated conditions, IRF can be measured if other competing channels such as dissociation or collisions do not deactivate the molecules. This process occurs on long molecular timescales, up to seconds, and is ubiquitous in space. In the laboratory, accessible time-scales are usually shorter but still sufficient to detect IRF. Thus, in principle, laser induced fluorescence can be applied by monitoring the IR instead of the traditional optical range of wavelengths. Benzene has been used as a prototype for this form of spectroscopy and in particular the region at the onset of the so-called channel 3 in $\mathrm{S}_{1} \leftarrow \mathrm{S}_{0}$ transition (see Feraud et al. (2011) for more details). Extension of this method to larger PAHs will occur in the future.

\subsection{Anomalous or Poincaré fluorescence}

As long as inverse internal conversion is possible, emission that does not follow Kasha's rule can be observed (Itoh 2012). To make such a process efficient, and to detect emission from excited states to lower states, including the ground state, small energy gaps between electronic states and large oscillator strengths are necessary. Such a process is indeed far from being rare, although experimental evidence is difficult to obtain in the gas phase, as is the case for pyrene (Numata et al. 2012). Recent experiments in which ionic PAHs have been trapped in an ion storage ring provide experimental evidence for an additional radiative relaxation channel (Martin et al. 2013). The deduced rate is a few hundreds $\mathrm{s}^{-1}$, compatible with the Poincaré fluorescence and clearly in competition with the IRF. Ideally, such experiments should be repeated with photon detection. These emissions may open the route to the study of the electronic spectroscopy of large ions in the gas phase by laser induced fluorescence.

\section{Conclusion}

Spectroscopic investigations of PAHs are continuing across the near-IR to the UV spectral range, with investigations of new classes of molecules (radicals and ions), longlived states at high resolution and short-lived states with broad absorptions. The ongoing development of different chemical reactors is opening the route to new PAH structures and larger species. Available gas-phase PAH spectra have been compared to astronomical data, so far with no convincing correlations. This does not rule out the PAH hypothesis because many possibilities remain to be tested. Refining and extending our understanding of the stability of the interstellar PAHs and their expected structures is one of the main goals for future research.

\section{Acknowledgements}

The authors would like to thank Ch. Jouvet and C. Dedondert-Lardeux for helpul discussions.

\section{References}

Alata, I., Bert, J., Broquier, M., Dedonder, C., Feraud, G., Gregoire, G., Soorkia, S., Marceca, E., \& Jouvet, Ch. 2013, J. Phys. Chem. A, 117, 4420

Alata, I., Broquier, M., Dedonder, C., Jouvet, C., \& Marceca, E. 2012, Chem. Phys., 393, 25

Alata, I., Dedonder, C., Broquier, M., Marceca, E., \& Jouvet, Ch. 2010a, J. Am. Chem. Soc., 132,17483 
Alata, I., Omidyan, R., Broquier, M., et al. 2010b, Phys. Chem. Chem. Phys., 12, 14456

Allamandola, L., Tielens, A., \& Barker, J. 1985, ApJ Letters, 290, L25

Baba, M., Kowaka, Y., Nagashima, U., Ishimoto, T., Goto, H., \& Nakayama, N. 2011, J. Chem. Phys., 135, 054305

Biennier, L., Salama, F., Allamandola, L. J., \& Scherer, J. J. 2003, J. Chem. Phys., 118, 7863

Brumfield, B. E., Stewart, J. T., \& McCall, B. J. 2012, J. Phys. Chem. Lett., 3, 1985

Carpentier, Y., Pino, T., \& Bréchignac, Ph. 2013, J. Phys. Chem. A, 117, 10092

Chakraborty, S., Omidyan, R., Alata, I., et al. 2009, J. Am. Chem. Soc., 131, 11091

Chalyavi, N., Dryza, V., Sanelli, J. A., \& Bieske, E. J. 2013, J. Chem. Phys., 138, 224307

Chalyavi, N., Troy, T. P., Bacskay, G. B., Nauta, K., Kable, S. H., Reid, S. A., \& Schmidt, T. W. 2012, J. Phys. Chem. A, 116, 10780

Chalyavi, N., Troy, T. P., Nakajima, M., Gibson, B. A, Nauta, K., Sharp, R. G., Kable, S. H., \& Schmidt, T. W 2011, J. Phys. Chem. A, 115, 7959

Coulson, C. A. \& Rushbrooke, G. S. 1940, Proc. Cambridge Phil. Soc., 36, 193

Dryza, V., Chalyavi, N., Sanelli, J. A., \& Bieske, E. J. 2012, J. Chem. Phys., 137, 204304

Dryza, V., Sanelli, J., Robertson, E., \& Bieske, E. 2012, J. Phys. Chem. A, 116, 4323

Feraud, G., Carpentier, Y., Pino, T., et al. 2013, in EAS Publications Series, Vol. 58, eds. Stehle, C., Joblin, C., \& d'Hendecourt, L., (EDP Sciences), 379-384

Fukushima, M. \& Obi, K. 1990, J. Chem. Phys., 93, 8488

Garkusha, I., Fulara, J., \& Maier, J. P. 2012,J. Mol. Struct., 1025,147

Garkusha, I., Fulara, J., Nagy, A., \& Maier, J. P. 2011,ApJ, 728,

Garkusha, I., Fulara, J., Sarre, P. J., \& Maier, J. P. 2011, J. Phys. Chem. A, 115, 10972

Garkusha, I., Nagy, A., Fulara, J., et al. 2013, J. Phys. Chem. A, 117, 351

Heavan, M., Dimauro, L., \& Miller, T. 1983,Chem. Phys. Lett., 95, 347

Friha, H., Feraud, G., Pino, T., Parneix P., Dhaouadi Z., \& Brchignac, Ph. 2013, in EAS Publications Series, Vol. 58, ed. Stehle, C and Joblin, C and d'Hendecourt, L,(EDP Sciences), 373-78

Itoh, T. 2012, Chem. Rev., 112, 4541,

Kokkin, D. L., Troy, T. P., Nakajima, M., et al. 2008,ApJ Letters, 681, L49

Kowaka, Y., Suganuma, Y., Ashizawa, N., Goto, H., Ishimoto, T., Nagashima, U., \& Baba, M. 2010, J. Molec. Spectrosc., 260, 72

Kowaka, Y., Nakayama, N., Ishimoto, T., Nagashima, U., Yamanaka, T., Ozawa, N., \& Baba, M. 2012, Chem. Phys., 400, 178

Kowaka, Y., Yamanaka, T., \& Baba, M. 2012, J. Chem. Phys. 136, 154301

Krechkivska, O, Liu, Y., Lee, K., Nauta, K., Kable, S. H., \& Schmidt, T. W. 2013, submitted

Léger, A. \& Puget, J. 1984, A\&3A, 137,

Longuet-Higgins, H. \& Pople, J. 1955, Proc. Phys. Soc. London Sec. A, 68, 591

Martin, S., Bernard, J., Bredy, R., et al. 2013, Phys. Rev. Lett., 110, 063003

Nagy, A., Fulara, J., \& Maier, J. P. 2011, J. Am. Chem. Soc., 133, 19796

Numata, Y., Suzuki, Y., \& Suzuka, I. 2012, J. Photochem. Photobio. A-Chem., 237, 49

O'Connor, G. D., Troy, T. P., Roberts, D. A., Chalyavi, N., Fückel, B., Crossley, M., Nauta, K., Stanton, J. F., \& Schmidt, T. W. 2011, J. Am. Chem. Soc., 133, 14554

O'Connor, Bacskay, Woodhouse, 2013, J. Phys. Chem. A, in press

Pino, T., Boudin, N., \& Brechignac, Ph. 1999, J. Chem. Phys., 111, 7337

Pino, T., Carpentier, Y., Feraud, G., et al. 2011, in EAS Publications Series, Vol. 46, PAHS and the Universe: a symposium to celebrate the 25th anniversary of the PAH hypothesis, ed. Joblin, C and Tielens, AGGM, (EDP Sciences), 355-371

Reilly, N. J., Kokkin, D. L., Nakajima, M., Nauta, K., Kable, S. H., \& Schmidt, T. W. 2008, J. Am. Chem. Soc., 130, 3137

Rode, M. F., Sobolewski, A. L., Dedonder, C., Jouvet, C., \& Dopfer, O. 2009,J. Phys. Chem. $A, 113,5865$

Salama, F. 2007, "Molecules in Space and Laboratory", J. L. Lemaire \& F. Combes, eds., Paris.

Salama, F. \& Allamandola, L. 1991,J. Chem. Phys.,94, 6964

Simpson, C., Brand, J., Berresheim, A., et al. 2002, Chemistry- Eur. J., 8, 1424 
Suganuma, Y., Kowaka, Y., Ashizawa, N.. Nakayama, N., Goto, H., Ishimoto, T., Nagashima, U., Ueda, T., Yamanaka, T., Nishi, N., \& Baba, M. 2011, Mol. Phys., 109, 1831

Tokmachev, A. M., Boggio-Pasqua, M., Mendive-Tapia, D., Bearpark, M. J., \& Robb, M. A. 2010,J. Chem. Phys., 132

Troy, T. P., Nakajima, M., Chalyavi, N., Clady, R. G. C. R., Nauta, K., Kable, S. H., \& Schmidt, T. W. 2009, J. Phys. Chem. A, 113, 10279

Troy, T. P. , Chalyavi, N., Menon, A. S., O’Connor, G. D., Fückel, B., Nauta, K, Radom, L., \& Schmidt, T. W. 2011, Chem. Sci., 2, 1755

Woodhouse, G. V. G. 2012, Honours Dissertation, School of Chemistry, University of Sydney 